TITLE:

\title{
Single-Shot Femtosecond Electron Diffraction with Laser-Accelerated Electrons: Experimental Demonstration of Electron Pulse Compression
}

\section{AUTHOR(S):}

Tokita, Shigeki; Hashida, Masaki; Inoue, Shunsuke; Nishoji, Toshihiko; Otani, Kazuto; Sakabe, Shuji

\section{CITATION:}

Tokita, Shigeki ...[et al]. Single-Shot Femtosecond Electron Diffraction with LaserAccelerated Electrons: Experimental Demonstration of Electron Pulse Compression. Physical Review Letters 2010, 105(21): 215004.

\section{ISSUE DATE:}

2010-11

URL:

http://hdl.handle.net/2433/131847

RIGHT:

(C) 2010 The American Physical Society 


\title{
Single-Shot Femtosecond Electron Diffraction with Laser-Accelerated Electrons: Experimental Demonstration of Electron Pulse Compression
}

\author{
Shigeki Tokita, ${ }^{*}$ Masaki Hashida, Shunsuke Inoue, Toshihiko Nishoji, Kazuto Otani, and Shuji Sakabe \\ Advanced Research Center for Beam Science, Institute for Chemical Research, Kyoto University, \\ Gokasho, Uji, Kyoto 611-0011, Japan \\ and Department of Physics, Graduate School of Science, Kyoto University, Kitashirakawa, Sakyo, Kyoto 606-7501, Japan
}

(Received 15 July 2010; published 17 November 2010)

\begin{abstract}
We report the first experimental demonstration of longitudinal compression of laser-accelerated electron pulses. Accelerated by a femtosecond laser pulse with an intensity of $10^{18} \mathrm{~W} / \mathrm{cm}^{2}$, an electron pulse with an energy of around $350 \mathrm{keV}$ and a relative momentum spread of about $10^{-2}$ was compressed to a $500-\mathrm{fs}$ pulse at a distance of about $50 \mathrm{~cm}$ from the electron source by using a magnetic pulse compressor. This pulse was used to generate a clear diffraction pattern of a gold crystal in a single shot. This method solves the space-charge problem in ultrafast electron diffraction.
\end{abstract}

DOI: 10.1103/PhysRevLett.105.215004

PACS numbers: $52.38 . \mathrm{Kd}, 61.05 . J-$

With femtosecond electron pulses, ultrafast electron diffraction (UED) [1-4] and x-ray pulse generation in free-electron lasers [5] are essential for investigating the ultrafast structural dynamics of atomic and molecular systems through time-resolved structural analysis. There are two typical methods for generating femtosecond electron pulses. For high energy applications such as free-electron lasers and megaelectronvolt ultrafast electron diffraction (MeV UED) [6], rf accelerators are used to produce a high acceleration field and to control the longitudinal beam dynamics. For energies higher than a few megaelectronvolts, high-brightness femtosecond pulses with a charge of more than $1 \mathrm{pC}$ can be produced without significant pulse stretching caused by space-charge forces. On the other hand, for low-energy applications such as conventional UED, dc acceleration with a voltage of tens of kilovolts is commonly used. With conventional instruments, an electron pulse is generated at a photocathode irradiated by femtosecond laser pulses and accelerated by an external static electric field. The longitudinal beam dynamics are not artificially controlled, and thus the electron pulse expands during its flight because of the space-charge forces in the pulse. As a result, the charge in a pulse is limited to the order of only $1 \mathrm{fC}$ in order to maintain short pulse duration [7-9]. This is the most important problem in current UED because it is impossible to perform singleshot measurements with such a low-brightness electron pulse. It is absolutely imperative to increase the charge in a UED pulse in order to study wide-ranging ultrafast phenomena involving many irreversible processes. Furthermore, for the midenergy range of around $100 \mathrm{keV}$ to $1 \mathrm{MeV}$, corresponding to the energies of conventional transmission electron microscopy (TEM), there is no satisfactory method for generating femtosecond electron pulses. The method of dc acceleration, for example, produces an extremely low-charge pulse that contains only one or a few electrons [10].
Recently, low-energy and midenergy femtosecond electron sources that overcome the space-charge problem in UED have been proposed and are under development [11-13]. A common principle of these methods is the longitudinal compression of the nonmonoenergetic electron pulses after dc acceleration. An alternative method that we have proposed is the use of laser acceleration and pulse compression [14]. In this method, electrons are accelerated by interaction of an ultraintense laser field with solid-density plasma, producing a broad-energy femtosecond electron pulse in the midenergy range that has low longitudinal emittance. Such an electron pulse immediately expands in the longitudinal direction during its flight, but the expansion process can be reversed by using phase rotation techniques $[15,16]$. It is expected that these laserdriven electron sources will have advantages in terms of both compressibility and brightness when compared with conventional photocathode-based electron sources [14].

In this Letter, we report the experimental demonstration of femtosecond pulse compression of a laser-accelerated electron beam with energy of around $350 \mathrm{keV}$. The electron pulses are generated by irradiating a tightly focused terawatt femtosecond laser pulse on a solid target; then, the pulses are compressed by using an achromatic bending magnet system. These femtosecond electron pulses have an intensity that is sufficient to take a single-shot diffraction pattern.

We performed electron pulse compression and pulse duration measurement using the setup shown in Fig. 1. A laser beam from a Ti:sapphire chirped-pulse amplification system (central wavelength $800 \mathrm{~nm}$, pulse duration $150 \mathrm{fs}$, pulse energy $140 \mathrm{~mJ}$ ) is split into two half beams. One is used to generate the electrons, and the other is used to measure the temporal duration of electron pulses from the scattering of the electron beam by the ponderomotive force [17]. Each beam is focused to a waist of $6 \times 4 \mu \mathrm{m}$ in FWHM with an $f / 3.5$ off-axis parabolic mirror, resulting 
(a)

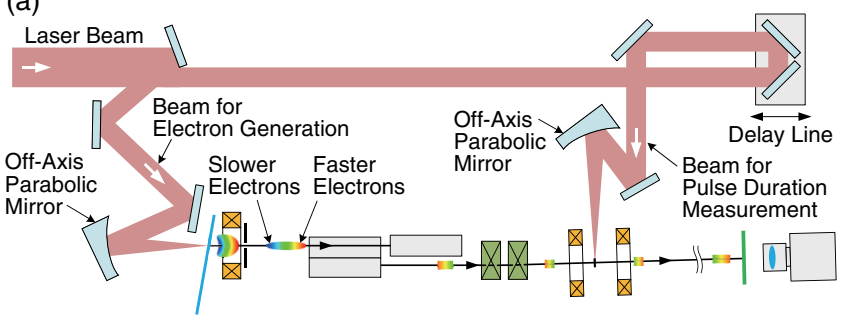

(b) Collimation Lens Dipole Magnets

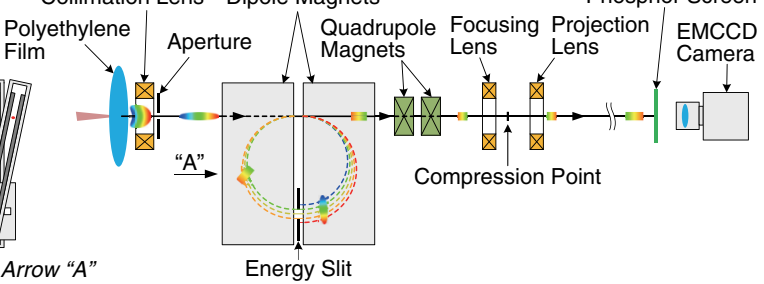

View on Arrow "A"

FIG. 1 (color online). Schematic diagram of the experimental setup of electron pulse compression and pulse duration measurement: (a) top view and (b) side view. Everything except the EMCCD camera is operated in a vacuum at $10^{-2} \mathrm{~Pa}$.

in an intensity of $\sim 10^{18} \mathrm{~W} / \mathrm{cm}^{2}$ at the beam waist. Amplified spontaneous emission is measured to be less than $10^{-7}$ of the peak intensity of the laser pulses. The generation beam irradiates a polyethylene film with a thickness of $10 \mu \mathrm{m}$, with $p$ polarization at an incidence angle of $10^{\circ}$ from the normal. Under these irradiation conditions, electrons are accelerated mainly by the $J \times B$ heating process [18]. It is observed that the electrons emitted from the back of the film have a broad angular distribution and a broad energy spectrum covering the range from $100 \mathrm{keV}$ to $1 \mathrm{MeV}$. A portion of the emitted electrons are collimated to a $\sim 350 \mathrm{keV}$ electron beam of $1 \mathrm{~mm}$ in diameter using an aperture and a permanent magnetic lens with a focal length of $\sim 15 \mathrm{~mm}$ at $350 \mathrm{keV}$. The first energy selection is performed in this stage, since most of the electrons, except those with energies of around $350 \mathrm{keV}$, are not collimated due to the chromatism of the lens. Pulse compression and further energy selection are accomplished by magnetic compression with dipole magnets [15] as follows. The electron beam is guided into two permanent dipole magnets, each of which gives a bending angle of $180^{\circ}$ and produces a uniform magnetic field of $63 \mathrm{mT}$ in a gap of $3 \mathrm{~mm}$. In the dipole magnets, electrons with higher energy follow a longer path than electrons with lower energy. Between the two magnets, a slit is placed to select a momentum width of $\sim 1 \%$ at $350 \mathrm{keV}$. The second magnet (the lefthand one in Fig. 1) is tilted at $12^{\circ}$ to the plane of the first so that the magnets themselves do not interfere with the electron beam emerging from the magnets. A pair of quadrupole magnets is used to correct the slight inherent chromatism of this dipole magnet system and to adjust the focus of the beam. To measure the temporal pulse duration, the electron beam is focused to $70 \mu \mathrm{m}$ at FWHM using a (a)

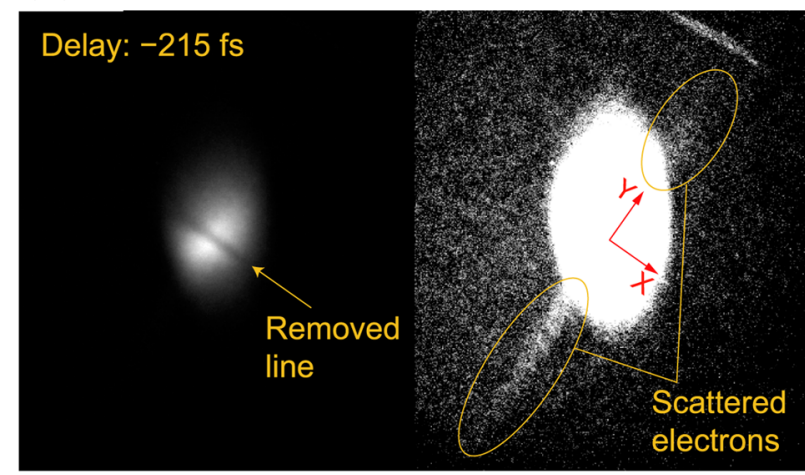

(b)

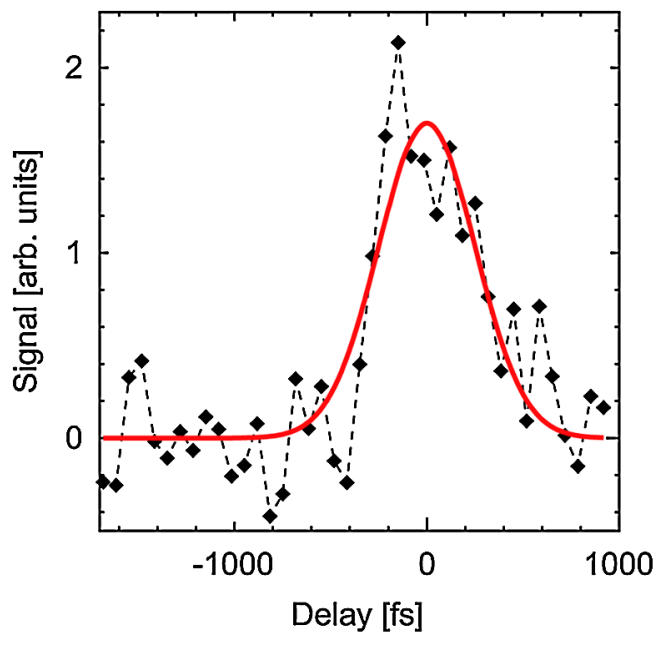

FIG. 2 (color online). (a) Typical image of an electron beam in which electrons are partially scattered by the ponderomotive force of the measurement laser beam, obtained in 20 shot acquisition. Both images are constructed from the same digital data but are displayed with different gray levels. (b) Signal trace of the scattered electrons with a Gaussian fit. FWHM of the Gaussian fit is $593 \pm 67$ fs.

permanent magnet focusing lens, and crosses the measurement laser beam. A transverse electron beam image at a distance of a few millimeters from the beam crossing point, which contains a scattering signal, is projected with a magnification factor of about 20 onto a phosphor screen using a permanent magnet projection lens and is detected by an electron-multiplying charge-coupled device (EMCCD) camera. The distance between the polyethylene film and the beam crossing point is $\sim 0.45 \mathrm{~m}$.

Figure 2(a) shows a typical image of an electron beam in which electrons are partially scattered by the measurement laser beam, where the delay between the laser pulse and the electron pulse is optimized to obtain a strong scattering signal. The removal of electrons along the direction of laser propagation is clearly observed in the electron beam cross section. The image is rotated by about $30^{\circ}$ on the screen due to the effect of the magnetic projection lens. As the pulse is more compressed the earlier it arrives at the compression point, the energy of the electron beam has been optimized by adjusting the position of the energy slit 
to minimize the arrival time of the electron pulse. To determine the temporal duration of electron pulses, we used the method proposed by Hebeisen et al. [17]. From measured images at various delay times, the ponderomotive scattering signals are numerically calculated using following equation:

$$
S(\tau)=\int|Y| D_{\tau}(X, Y) d X d Y
$$

where $X$ and $Y$ are the rectangular coordinates on the detector screen as shown in Fig. 2(a) and $D_{\tau}(X, Y)$ is the number density of electrons detected in the image at delay time $\tau$. The $S(\tau)$ can be identified as a convolution of the temporal and spatial profiles of the laser pulse and the electron pulse as explained in Ref. [17]. A signal trace with Gaussian fit is shown in Fig. 2(b). Assuming that the spatial and temporal intensity distributions of the laser and the electron pulses are Gaussian, we can determine the pulse duration of the electron pulse to be $524 \pm 59$ fs at FWHM. The electric charge in a pulse that arrives at the phosphor screen is estimated to be $6 \mathrm{fC}$.

We carried out numerical simulations of the electron trajectories using the General Particle Tracer (GPT) code [19] in order to model the pulse compression experiments. The electron source produced at the laser focal spot is assumed to have uniform energy and angular distributions initially. The emitted electrons are assumed to have Gaussian distributions in space and time with a FWHM of $6 \times 4 \mu \mathrm{m}$ and a FWHM of $150 \mathrm{fs}$, respectively. The space-charge effect is initially ignored. Figure 3 shows the longitudinal phase-space and current distributions of the electron pulse at the compression point. The pulse duration and energy width are calculated to be $220 \mathrm{fs}$ and $7 \mathrm{keV}$ at FWHM. The increase from 150 to $220 \mathrm{fs}$ is mainly due to the collimation and focusing lenses. This result shows that the flight time difference in the magnet system is relatively small. Thus, the longer pulse obtained experimentally cannot be attributed to the performance of the pulse compression system. Further numerical simulations taking into account the space-charge forces confirm that the effect of those forces on the pulse duration is negligible in this experiment. We, therefore, conclude that the increased pulse duration is mainly due to the characteristics of the laser-driven electron source. However, the intrinsic time response of a laser-driven electron source with energy of hundreds of kiloelectronvolts has not been researched in detail. At present, we expect some effects from the preformed plasma produced by the amplified spontaneous emission pulse that accompanies the main intense laser pulse, as well as from the electron circulation in the plasma caused by the sheath electric fields, which are formed on both sides of the plane plasma surface [20]. In any case, we expect to be able to produce shorter electron pulses by improving the laser pulses or targets.

We have demonstrated single-shot electron diffraction using the compressed electron pulses on a 10-nm-thick single-crystal (001) Au foil sample. The sample is set on a copper mesh used for general TEM observations and is placed at the pulse compression point after removing the focusing lens, the projection lens, and the measurement laser beam. In this configuration, the magnet system has been designed to minimize the electron beam size on the screen; that is, the achromaticity of the system is maintained. The electron beam sizes at the sample and the screen are measured using an imaging plate and the EMCCD camera to be $560 \times 890 \mu \mathrm{m}$ and $430 \times$ $280 \mu \mathrm{m}$ at FWHM, respectively. The distance between the sample and the screen is $0.41 \mathrm{~m}$. Figure 4(a) shows a single-shot diffraction pattern for the Au sample. The peak intensities of the diffraction spots for the (020), (200), and (220) planes are sufficiently strong to be detected. For example, the (020) spot intensity is about 40 times higher than the rms background noise of the image. With multiple-shot measurement, the clarity of the image is improved: as shown in Fig. 4(b), second-order diffraction spots became visible with 10-shot accumulation. From the distances between the diffraction spots and the known lattice parameters of $\mathrm{Au}$, the central electron energy is determined to be $356 \pm 7 \mathrm{keV}$.

We have demonstrated that femtosecond laser plasma can serve as a high-brightness electron source providing a self-compressed femtosecond electron pulse with energy of hundreds of kiloelectronvolts. This technique has great potential for the generation of extremely high-charge

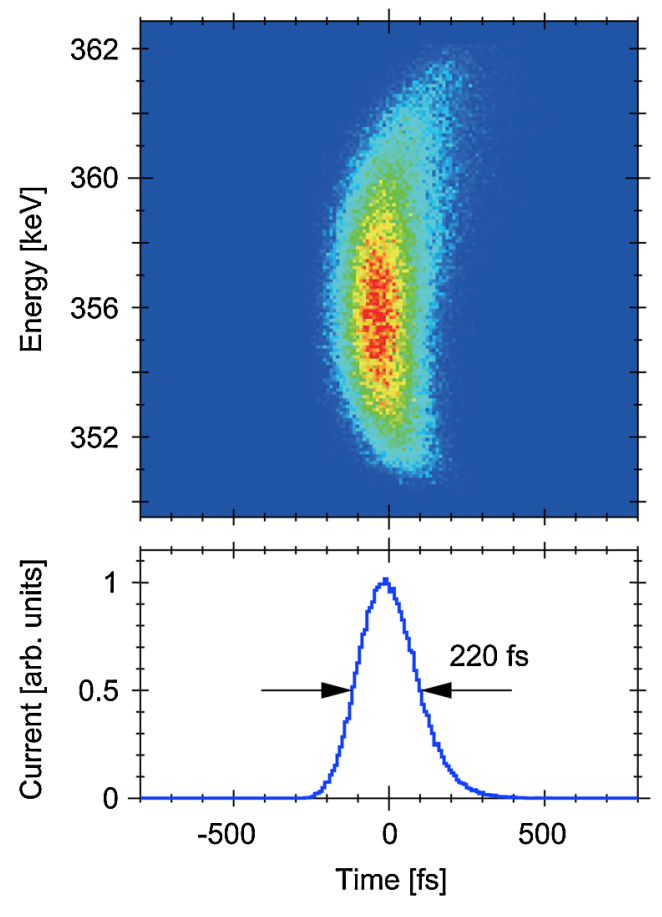

FIG. 3 (color online). Longitudinal phase-space distribution and current distribution in numerical simulations. FWHM of the current distribution and FWHM of the energy width are $220 \mathrm{fs}$ and $7 \mathrm{keV}$, respectively. 


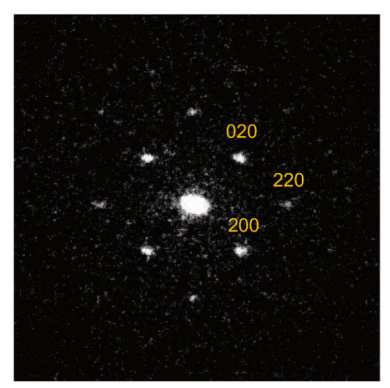

(a)

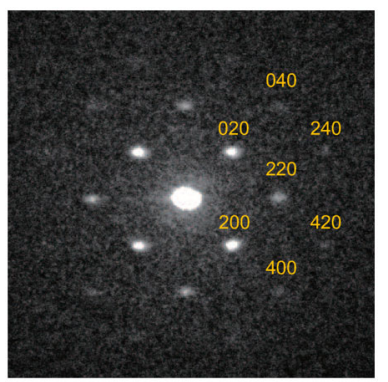

(b)
FIG. 4 (color online). Diffraction patterns obtained from $\mathrm{Au}$ (001) single-crystal sample with (a) single-shot and (b) 10-shot irradiation.

femtosecond pulses in the midenergy range, because the space-charge effect does not limit the charge in a pulse. There is also the possibility of further improvement in the brightness of the laser-driven electron source [14]. Moreover, since the compression system does not produce timing jitter between the electron pulse and the laser pulse, it is possible to obtain high temporal resolution in pumpand-probe experiments. We believe that this development will fundamentally change the observation of irreversible ultrafast phenomena by UED in the near future. Furthermore, this is a general technique that can be utilized for any application that needs femtosecond probing or driving.

The authors thank Professor S. Isoda, Professor H. Kurata, and Professor T. Nemoto for their valuable suggestions. This work was supported by a Grant-in-Aid for Scientific Research (A) (Grant No. 18206006), a Grantin-Aid for Young Scientists (B) (Grant No. 20760032 and 22760038), and a Grant-in-Aid for the Global COE Program "The Next Generation of Physics, Spun from Universality and Emergence" from the Ministry of Education, Culture, Sports, Science and Technology (MEXT), Japan.

*To whom correspondence should be addressed. tokita@laser.kuicr.kyoto-u.ac.jp

[1] R. Srinivasan, V. A. Lobastov, C.-Y. Ruan, and A.H. Zewail, Helv. Chim. Acta 86, 1761 (2003).

[2] W. E. King, G. H. Campbell, A. Frank, B. Reed, J. F. Schmerge, B. J. Siwick, B. C. Stuart, and P. M. Weber, J. Appl. Phys. 97, 111101 (2005).

[3] J. R. Dwyer, C. T. Hebeisen, R. Ernstorfer, M. Harb, V. B. Deyirmenjian, R.E. Jordan, and R.J.D. Miller, Phil. Trans. R. Soc. A 364, 741 (2006).
[4] A.H. Zewail, Annu. Rev. Phys. Chem. 57, 65 (2006).

[5] A. M. Lindenberg, J. Larsson, K. Sokolowski-Tinten, K. J. Gaffney, C. Blome, O. Synnergren, J. Sheppard, C. Caleman, A. G. MacPhee, D. Weinstein, D. P. Lowney, T. K. Allison, T. Matthews, R.W. Falcone, A.L. Cavalieri, D. M. Fritz, S. H. Lee, P. H. Bucksbaum, D. A. Reis, J. Rudati, P. H. Fuoss, C. C. Kao, D. P. Siddons, R. Pahl, J. Als-Nielsen, S. Duesterer, R. Ischebeck, H. Schlarb, H. Schulte-Schrepping, Th. Tschentscher, J. Schneider, D. von der Linde, O. Hignette, F. Sette, H. N. Chapman, R. W. Lee, T. N. Hansen, S. Techert, J. S. Wark, M. Bergh, G. Huldt, D. van der Spoel, N. Timneanu, J. Hajdu, R. A. Akre, E. Bong, P. Krejcik, J. Arthur, S. Brennan, K. Luening, and J. B. Hastings, Science 308, 392 (2005).

[6] J. B. Hastings, F. M. Rudakov, D. H. Dowell, J. F. Schmerge, J. Cardoza, J. M. Castro, S. M. Gierman, H. Loos, and P. M. Weber, Appl. Phys. Lett. 89, 184109 (2006).

[7] M. Dantus, S. B. Kim, J. C. Williamson, and A. H. Zewail, J. Phys. Chem. 98, 2782 (1994).

[8] J. Cao, Z. Hao, H. Park, C. Tao, D. Kau, and L. Blaszczyk, Appl. Phys. Lett. 83, 1044 (2003).

[9] C. T. Hebeisen, G. Sciaini, M. Harb, R. Ernstorfer, T. Dartigalongue, S. G. Kruglik, and R.J.D. Miller, Opt. Express 16, 3334 (2008).

[10] M. S. Grinolds, V. A. Lobastov, J. Weissenrieder, and A. H. Zewail, Proc. Natl. Acad. Sci. U.S.A. 103, 18427 (2006).

[11] P. Baum and A. H. Zewail, Proc. Natl. Acad. Sci. U.S.A. 103, 16105 (2006).

[12] T. van Oudheusden, E.F. de Jong, S. B. van der Geer, W.P. E. M. Op 't Root, O. J. Luiten, and B. J. Siwick, J. Appl. Phys. 102, 093501 (2007).

[13] G. H. Kassier, K. Haupt, N. Erasmus, E. G. Rohwer, and H. Schwoerera, J. Appl. Phys. 105, 113111 (2009).

[14] S. Tokita, S. Inoue, S. Masuno, M. Hashida, and S. Sakabe, Appl. Phys. Lett. 95, 111911 (2009).

[15] P. Kung, H. C. Lihn, H. Wiedemann, and D. Bocek, Phys. Rev. Lett. 73, 967 (1994).

[16] S. B. van der Geer, M.J. de Loos, T. van Oudheusden, W.P.E.M. Op 't Root, M.J. van der Wiel, and O.J. Luiten, Phys. Rev. ST Accel. Beams 9, 044203 (2006).

[17] C. T. Hebeisen, R. Ernstorfer, M. Harb, T. Dartigalongue, R.E. Jordan, and R.J.D. Miller, Opt. Lett. 31, 3517 (2006).

[18] W. L. Kruer and K. Estabrook, Phys. Fluids 28, 430 (1985).

[19] General Particle Tracer code, http://www.pulsar.nl/gpt/.

[20] Y. Sentoku, T.E. Cowan, A. Kemp, and H. Ruhl, Phys. Plasmas 10, 2009 (2003). 